\title{
Risk factors for wound complications after ankle fracture surgery.
}

Adam G Miller

Department of Orthopedics, Thomas Jefferson University Hospital

Andrew Margules

Department of Orthopedics, Thomas Jefferson University Hospital

Steven M Raikin

Rothman Institute, Thomas Jefferson University

Follow this and additional works at: https://jdc.jefferson.edu/orthofp

Part of the Orthopedics Commons

Let us know how access to this document benefits you

\section{Recommended Citation}

Miller, Adam G; Margules, Andrew; and Raikin, Steven M, "Risk factors for wound complications after ankle fracture surgery." (2012). Department of Orthopaedic Surgery Faculty Papers. Paper 45.

https://jdc.jefferson.edu/orthofp/45

This Article is brought to you for free and open access by the Jefferson Digital Commons. The Jefferson Digital Commons is a service of Thomas Jefferson University's Center for Teaching and Learning (CTL). The Commons is a showcase for Jefferson books and journals, peer-reviewed scholarly publications, unique historical collections from the University archives, and teaching tools. The Jefferson Digital Commons allows researchers and interested readers anywhere in the world to learn about and keep up to date with Jefferson scholarship. This article has been accepted for inclusion in Department of Orthopaedic Surgery Faculty Papers by an authorized administrator of the Jefferson Digital Commons. For more information, please contact: JeffersonDigitalCommons@jefferson.edu. 


\title{
Risk Factors for Wound Complications After Ankle Fracture Surgery
}

\author{
Adam G. Miller, MD, Andrew Margules, MD, and Steven M. Raikin, MD \\ Investigation performed at the Rothman Institute of Orthopedics, Thomas Jefferson University Hospital, Philadelphia, Pennsylvania
}

\begin{abstract}
Background: The overall rate of complications after ankle fracture fixation varies between $5 \%$ and $40 \%$ depending on the population investigated, and wound complications have been reported to occur in $1.4 \%$ to $18.8 \%$ of patients. Large studies have focused on complications in terms of readmission, but few studies have examined risk factors for woundrelated issues in the outpatient setting in a large number of patients. A review was performed to identify risk factors for wound complications tracked in the hospital and outpatient setting.
\end{abstract}

Methods: Four hundred and seventy-eight patients underwent open reduction and internal fixation of an ankle fracture between 2003 and 2010 by a single surgeon at a single institution. Demographic characteristics, time to surgery, comorbidities, and postoperative care were tracked. Wound complications were defined as those requiring dressing care and oral antibiotics or requiring further surgical treatment.

Results: Of the 478 patients who were followed, six (1.25\%) had wounds requiring surgical debridement. Fourteen patients $(2.9 \%)$ required further dressing care or a course of oral antibiotics. There were significant associations between wound complications and a history of diabetes $(p<0.001)$, peripheral neuropathy $(p=0.003)$, wound-compromising medications $(p=0.011)$, open fractures $(p=0.05)$, and postoperative noncompliance $(p=0.027)$. There was a significant difference in age between patients with and without wound complications $(p=0.045)$. We did not identify a relationship between time to surgery and complications.

Conclusions: These results highlight the difficulty of treating medically complex and noncompliant patient populations. With careful preoperative monitoring of swelling, time to surgery does not affect wound outcome. The failure of the patient to adhere to postoperative instructions should be a concern to the treating surgeon.

Level of Evidence: Prognostic Level I. See Instructions for Authors for a complete description of levels of evidence.

A nkle fracture is a common injury, with a reported incidence of 184 per 100,000 person-years ${ }^{1}$. Most are stable fractures that are easily treated nonoperatively. Surgical treatment of the unstable ankle fracture generally is associated with improved outcomes, even in geriatric patients $\mathrm{s}^{2,3}$.

The overall rate of complications after open reduction and internal fixation of ankle fracture varies between $5 \%$ and $40 \%$, depending on the population investigated ${ }^{4}$. Analysis has most frequently suggested increased complications following open injuries in the elderly and diabetic subpopulations $s^{2,5-9}$. Wound complications after open reduction and internal fixa- tion of ankle fractures have been reported to occur in $1.4 \%$ to $18.8 \%$ of patients $\mathrm{s}^{10-12}$.

Most of the literature on wound complications is based on small series of patients. Large database studies have focused on complications only in terms of readmission to the hospital or return to the operating room as the primary outcomes. To our knowledge, no study has examined risk factors for woundrelated complications in the outpatient setting in a large number of patients. In the present study, we hypothesized that medical comorbidities identified preoperatively and open fractures would have a negative impact on the wound complication rate.

Disclosure: None of the authors received payments or services, either directly or indirectly (i.e., via his or her institution), from a third party in support of any aspect of this work. One or more of the authors, or his or her institution, has had a financial relationship, in the thirty-six months prior to submission of this work, with an entity in the biomedical arena that could be perceived to influence or have the potential to influence what is written in this work. No author has had any other relationships, or has engaged in any other activities, that could be perceived to influence or have the potential to influence what is written in this work. The complete Disclosures of Potential Conflicts of Interest submitted by authors are always provided with the online version of the article. 
The Journal of Bone \& Joint Surgery $\cdot$ JBjs.org Volume 94-A • Number 22 - November 21, 2012
Risk Factors for Wound Complications After Ankle Fracture Surgery

\section{Materials and Methods}

\section{Demographic Characteristics}

fter appropriate institutional review board approval had been obtained, a A retrospective database review was undertaken for ICD-9 (International Classification of Diseases, Ninth Revision) diagnosis codes for ankle fractures (824.0 to 824.9), with the exclusion of pilon fractures and matching CPT (Current Procedural Terminology) codes for surgical treatment corresponding to these codes $(27792,27814,27822,27823)$, with use of electronic medical records software (SRS EMR, version 7.3; SRSsoft, Montvale, New Jersey). The study population comprised all consecutive ankle fractures that were treated by a single surgeon (S.M.R.) at a single institution between January 2003 and December 2010; no cases were excluded. In all, 478 patients were identified and included in the study. There were no bilateral cases.

All documented clinical notes were reviewed for all cases. These included an initial preclinical questionnaire completed by the patient, the initial history and physical examination, operative reports, and all office notes for a minimum of three months postoperatively. All patients were followed until complete wound-healing had occurred.

The mean age of the patients was 43.2 years (range, twelve to ninety years; median, forty-four years). The cohort included 249 females (52.1\%). The average body mass index (BMI) was $29 \mathrm{~kg} / \mathrm{m}^{2}$. The demographic characteristics of the patients are summarized in Table I.

\section{Risk Factors}

The assessment of perioperative risk focused on variables previously described in literature as potentially increasing the likelihood of wound-related complications. These included (1) patient-related variables, including diabetes, peripheral vascular disease (PVD), HIV/AIDS, cigarette smoking, inflammatory diseases, the use of corticosteroids and other wound-healing-compromising medications ${ }^{13}$, BMI, age, sex, and previous surgery involving the injured ankle; (2) operative and injury-related factors, including the mechanism of injury, the time to surgery following the injury, the type of fracture (categorized as unimalleolar, bimalleolar equivalent, bimalleolar and trimalleolar, ankle dislocation, or syndesmosis injury), polytrauma, and open fracture; and (3) the postoperative variable of patient compliance with physician instructions. Noncompliance was documented if the patient deviated from standard postoperative care as defined by the senior surgeon. The surgeon personally reviewed these guidelines with each patient, and uniform instructions were provided to each individual postoperatively. All deviations in care were considered to be of equal weight.

\section{Treatment Protocol}

All fracture blisters were drained under strict sterile conditions and were covered with a Betadine (povidone-iodine)-impregnated dressing at the point

\section{TABLE I Patient Characteristics}

\begin{tabular}{|c|c|}
\hline Age* (yr) & 43 (12 to 90$)$ \\
\hline Female:male ratio (no. of patients) & 249:229 \\
\hline Body mass index* $\left(\mathrm{kg} / \mathrm{m}^{2}\right)$ & 29 (10.6 to 79.0$)$ \\
\hline Time to surgery* $(d)$ & $9.2(0$ to 31$)$ \\
\hline Side (left:right) (no. of ankles) & 224:254 \\
\hline $\begin{array}{l}\text { Type of fracture (open:closed) } \\
\text { (no. of ankles) }\end{array}$ & 9:469 \\
\hline Diabetes (no:yes) $\dagger$ (no. of patients) & $418: 46$ \\
\hline
\end{tabular}
documentation in the patient records. when first identified. A well-padded splint was applied, and the patient was given instructions for strict limb elevation to minimize swelling. Surgery was delayed until there was resolution of all blisters and the "wrinkle test" demonstrated wrinkling of the skin in the proposed incisional area when gently pinched between two fingers ${ }^{14}$.

Postoperatively, our protocol included strict elevation of the limb for the first two weeks after surgery. The initial surgical splint was left in place during this time period, with the patient being instructed to return for a splint change for any problems or discomfort. All patients were given a five-day course of prophylactic antibiotics (in addition to preoperative intravenous antibiotics) with use of a first-generation cephalosporin. Incisional healing was assessed at two weeks, and sutures were removed when the incision was determined to be adequately healed clinically. The patient was managed with a fracture boot, and early range of motion was allowed as soon as the incisions were healed. All patients were instructed to remain non-weight-bearing for at least six weeks. Diabetic patients with peripheral neuropathy were kept nonweight-bearing for twelve weeks. Progressive weight-bearing as tolerated was initiated in a fracture boot after six weeks if there was radiographic evidence of healing when adequate fracture healing was seen radiographically. Formal physical therapy was instituted at the six-week point, and the boot was discontinued after three months or at six months in patients with diabetic peripheral neuropathy. Protocol adjustments were made for wound or fracturehealing problems.

\section{Outcomes}

Categorization of wound complications was based on the treatment required to achieve resolution. Patients were stratified into three categories: no complications, minor complications, and major complications. The first category (no complications) represented normal wound-healing and suture or staple removal without additional measures. The second category (minor complications) included superficial infection, minor wound dehiscence, or delayed wound healing. These patients required outpatient wound care, including office or home-based debridements, local wound care, and/or oral antibiotics. The third category (major complications) included patients who required a return to the operating room for additional surgical treatment of wound complications. These interventions included any form of surgical irrigation and debridement, with or without removal of hardware. Multiple surgical interventions for one ankle fracture were noted but were considered as one major complication. In addition, any patient requiring vacuum-assisted wound closure was included in this group. All patients were seen at two weeks postoperatively. Sutures or staples were removed at this time. Subsequent visits were scheduled at six weeks and three months, with patients who had complications returning more often. All database reviews and data collection were undertaken by a research assistant independently of the surgeon who performed the operations.

\section{Statistical Methods}

The collected data were subjected to statistical analysis with all wound complications as the dependent variable. This analysis included both minor and major complications. Minor and major wound complications were also analyzed separately. Pearson chi-square analysis and the Fisher exact test were used to compare categorical variables of potentially associated risk factors. The minor complication group was compared with the no complication group, and the major complication group was compared with the combined minor complication and no complication group. Finally, the combined minor and major complication group was compared with the no complication group. A Bonferroni correction was applied for pairwise comparisons (Table II). Continuous variables were compared with the Student $t$ test. Significant risk factors $(\mathrm{p}<0.05)$ for total (major and minor) wound complications were then sought through binomial logistic regression. Each risk factor was analyzed in a separate regression model. A final multivariate regression model with backwards elimination was performed with the significant variables from the univariate analysis. The standard error, odds ratio, and confidence interval were calculated. Confidence intervals not including 1 were considered significant. 
The Journal of Bone \& Joint Surgery $\cdot$ JBJS.org Volume 94-A · Number $22 \cdot$ November 21,2012

Risk Factors for Wound Complications After

Ankle Fracture Surgery

TABLE II Wound Complication Risk Factors

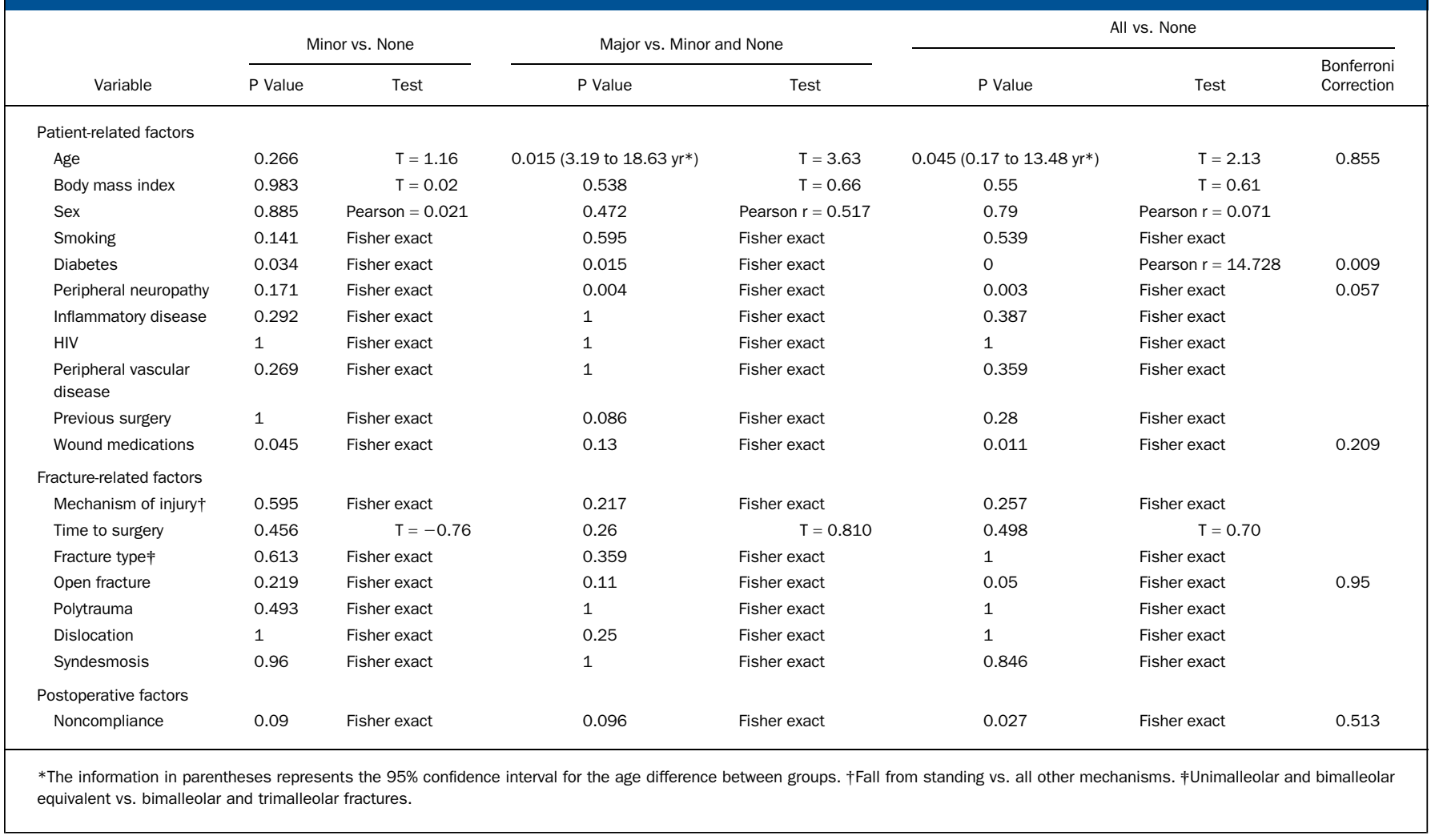

Delay to surgery was analyzed as both a continuous variable and as a categorical variable. For categorical analysis, the delay to surgery for each patient was transformed to a binary variable to establish a cutoff between early and late surgery. Cutoffs of three, five, seven, and ten days were analyzed.

\section{Source of Funding}

There was no source of funding for this project.

\section{Results}

$\mathrm{O}$ $\mathrm{f}$ the 478 ankle fractures undergoing open reduction and internal fixation, $458(95.8 \%)$ had wounds that healed completely without any further intervention. Fourteen ankles (2.9\%) had a minor complication (a complication requiring outpatient wound care and/or oral antibiotics to achieve complete resolution), whereas six ankles (1.25\%) had a major complication (a complication requiring additional surgical intervention).

Analysis of minor complications demonstrated significant associations between wound complications and a history of diabetes $(\mathrm{p}=0.034)$ or a history of taking medication that potentially affected wound-healing $(\mathrm{p}=0.045)$.

Patients with a major complication were older than those with no complications or a minor complication (mean age, 53.8 years versus 42.9 years; $\mathrm{p}=0.045$ ). Additionally, patients in the major complication group more often had a history of diabetes $(p=0.015)$. Peripheral neuropathy demonstrated an even stronger relationship with major complications $(\mathrm{p}=0.004)$.
When the patients with minor and major complications were compared with those with no complications, higher age remained a risk factor $(\mathrm{p}=0.045)$, as did a history of diabetes $(\mathrm{p}<0.001)$, a known peripheral neuropathy $(\mathrm{p}=0.003)$, a history of taking wound-healing-compromising medications $(\mathrm{p}=0.011)$, an open fracture $(\mathrm{p}=0.05)$, and postoperative noncompliance as documented in the patient charts $(\mathrm{p}=$ 0.027) (Table II).

Binomial logistic regression, performed on five significant variables, revealed that the odds of having a postoperative wound complication were increased 5.59 times in the setting of diabetes (with the peripheral neuropathy subgroup having a 15.49 times increased risk of wound-healing-related complications), 8.56 times in patients taking wound-healingcompromising medications, 7.03 times in patients with open fractures, and 3.63 times in patients who were noncompliant with physician instructions in the postoperative period (Table III). The odds of experiencing a wound complication with incrementally increasing patient age was 1.03 (95\% confidence interval, 1.00 to 1.06). The results of multivariate regression analysis are shown in Table IV.

Polytrauma to the limb undergoing ankle fracture fixation occurred in sixty-seven $(14.0 \%)$ of the 478 patients, but only two of these sixty-seven patients developed minor wound complications. Other injury-related factors such as dislocation or concomitant syndesmotic injury did not appear to be associated with an increased rate of wound complications. 
The Journal of Bone \& Joint Surgery $\cdot$ Jbjs.org Volume 94-A • Number 22 - November 21, 2012
Risk Factors for Wound Complications After ANKLE Fracture Surgery

TABLE III Regression Analysis: Calculation of Odds of Having Any Wound Complication

\begin{tabular}{|lccccc|}
\hline \multicolumn{1}{|c}{ Variable } & Standard Error & Odds Ratio & 95\% Confidence Interval & P Value \\
\hline Diabetes & 0.2817 & 5.59 & 2.11 to 14.84 & 0.002 \\
Peripheral neuropathy & 0.771 & 15.49 & 3.42 to 70.22 & 0.003 \\
Medication & 0.7137 & 8.56 & 2.11 to 34.68 & 0.003 \\
Open fracture & 0.837 & 7.03 & 1.36 to 36.27 & 0.02 \\
Noncompliance & 0.5435 & 3.63 & 1.25 to 10.54 & 0.018 \\
\hline
\end{tabular}

TABLE IV Multivariate Regression Analysis

\begin{tabular}{|lcccc|}
\hline \multicolumn{1}{|c}{ Variable } & Standard Error & Odds Ratio & 95\% Confidence Interval & P Value \\
\hline Diabetes & 0.5676 & 3.61 & 1.19 to 10.97 & 0.024 \\
Medication & 0.7519 & 6.75 & 1.55 to 29.47 & 0.011 \\
Open fracture & 1.010 & 13.43 & 1.86 to 97.18 & 0.010 \\
Noncompliance & 0.5964 & 3.66 & 1.14 to 11.77 & 0.030 \\
\hline
\end{tabular}

Also, the analysis of our cohort showed no correlation between patient-reported smoking and wound complications following ankle fracture surgery.

\section{Discussion}

$\mathrm{M}$ any studies have examined the outcomes of surgically treated ankle fracture $\mathrm{e}^{2-6,8-12,15-21}$. Generally, good results follow surgical intervention, and risk factors for poor outcomes and complications have been identified ${ }^{2,5-9}$. Wound complications comprise an important category of these complications. They usually occur in the early postoperative period, yet a persistent deep infection may ultimately affect the long-term function of the ankle. Few studies have specifically focused on wound complications, and those studies have been limited by small size $e^{2}$ or the use of short-term hospital outcome measures only $^{3,9}$. Our study combines outpatient follow-up and a large number of patients to counter these previous shortcomings.

SooHoo et al. performed an analysis of complications in all patients undergoing surgical treatment of ankle fractures in the state of California who required readmission or additional surgery on the ankle 9 . They reported that the rate of woundrelated complications was $1.44 \%$. In our study cohort, $1.25 \%$ of 478 patients required return to the operating room for woundrelated issues. SooHoo et al., however, did not review the rate of minor wound complications that did not require hospital readmission or further surgical treatment.

In the present study, we found that patients with diabetes (and particularly those with peripheral neuropathy), patients taking wound-compromising medications, patients who sustained open fractures, and patients who were not compliant postoperatively had an increased risk of wound-healing problems. In a recent small series of twenty-six patients with major postoperative infections after surgical treatment of ankle fractures, $81 \%$ of the infections occurred in a medically compromised host ${ }^{14}$; diabetes was the most frequently found compromising condition. Flynn et al. found that the risk of infection in diabetic patients with closed ankle fractures was $32 \%{ }^{5}$. Wukich et al. analyzed postoperative infection rates in diabetic patients and found that those who had peripheral neuropathy were at five times the risk of infection in comparison with controls ${ }^{16}$. We also found this subpopulation to be particularly prone to wound problems, with an odds ratio of 15.49 following univariate analysis.

The infection rate following the surgical treatment of open ankle fractures has been reported to be between $6 \%{ }^{17}$ and $40 \%{ }^{18}$. For patients with open fractures who present initially to our emergency department, forty-eight hours of antibiotic coverage is begun, with the type of antibiotic depending on the specific injury and patient allergies. In the present study, all patients underwent an initial irrigation and debridement plus internal fixation within six hours after presentation. All Grade3 open wounds subsequently were treated with a second irrigation and debridement after forty-eight hours. The nine open ankle fractures included two Grade-3A open fractures, five Grade- 2 open fractures, and two Grade-1 open fractures. Two of these nine fractures were associated with the development of an infection requiring further surgical intervention. This group of patients had the largest odds ratio in the multivariate analysis (13.43). From our series, it is unclear how to make improvements to our protocol, considering the relative success of this strategy reported in the literature in comparison with delayed internal fixation ${ }^{22,23}$. A recent meta-analysis of 498 open ankle fractures demonstrated that the rate of satisfactory results was $81 \%$ in the group treated with immediate internal fixation and $76 \%$ in the group treated with staged external fixation and delayed internal fixation ${ }^{24}$. The most frequently reported complications were deep infection $(8 \%)$ and skin necrosis (14\%). 
The Journal of Bone \& Joint Surgery · Jbjs.org Volume 94-A · Number 22 - November 21, 2012
Risk Factors for Wound Complications After Ankle Fracture Surgery
One variable that is often overlooked is the noncompliant patient. One previous article demonstrated that noncompliance was a risk for complications following ankle fracture surgery ${ }^{25}$, and the noncompliant diabetic patient has been described as the most difficult type of patient to treat ${ }^{5}$. In our cohort, thirty-eight patient records documented some degree of noncompliance during the early postoperative period, including failure to follow weight-bearing instructions, premature removal of splints, or failure to adhere to woundcare instructions. Five of these patients experienced wound problems.

Patient age has also been identified as a risk factor for postoperative complications after ankle fracture surgery, with complication rates as high as $25 \%^{2,9}$. The patients in the present study were younger, with an average age of forty-three years, and thus we could not identify age as a specific risk factor.

Some studies have demonstrated that the delay to surgery is a risk factor for wound complications, whereas other studies have demonstrated no effect ${ }^{19-21}$. The timing of surgery was not associated with wound complications in our study. While there was an increase in wound complications for patients who underwent surgery within five days $(6.15 \%)$ as compared with those who underwent surgery after the five-day mark (3.81\%), this difference was not significant $(\mathrm{p}=0.384)$. We attribute this to detailed examination of the soft-tissue envelope initially and before surgery. If the soft tissues were thought to be potentially compromised, the surgery was postponed. We prefer to delay surgical treatment for seven to fourteen days to allow soft-tissue swelling to resolve whenever possible, and the average time to surgery in this study was nine days (range, zero to thirty-one days). Patients were repeatedly instructed on the importance of elevation of the affected extremity above the heart level both preoperatively and postoperatively to minimize swelling and tension on the wound edges.

Cigarette smoking also has been reported as a major risk factor for poor outcomes following ankle fracture surgery ${ }^{26}$. Seventy-six of our patients reported on their intake questionnaire that they smoked cigarettes. All of these patients were counseled at the initial visit, on the day of surgery, and at each subsequent visit about the risks of smoking and nicotine use for wound-healing and fracture-healing. Patients were additionally advised to discuss a smoking-cessation program with their internist during their preoperative visit. In our study, only four smokers $(5.3 \%)$ developed a minor wound complication and no smoker developed a major wound complication, and thus smoking was not found to be a significant risk factor. We hypothesize that our results related to smoking may be due to the described counseling and potentially less nicotine use/cigarette smoking during the healing period. However, we cannot make any conclusions regarding smoking and the association with wound complications from the data that we analyzed.

There are obvious limitations to this study. While the present study represents the largest analysis of patients undergoing surgical treatment of ankle fractures who underwent direct individual follow-up, the numbers may still be too small to accurately assess each specific risk factor. Risk factors and their associations are limited to the accuracy with which patients report their medical history, and some risk factors may be underreported. Patients may not share some information that appears to them to be irrelevant at the time of the office visit preoperatively. However, data were collected from a database maintained by an independent research assistant, thereby eliminating recall bias on the part of the authors. In addition, there was no way to assess whether patients altered their risk factors during the perioperative period (smoking cessation, discontinuation of medications, better diabetes control, etc.). Finally, we applied a Bonferroni correction that resulted in statistical significance only for diabetes as a risk factor in the pairwise comparison analysis. While increasing the strictness of the analysis in this way may diminish the rate of false-positive results, we believe that this is outweighed by not missing the false-negative results that may lead a surgeon to underestimate the potential risk for wound complications that their patients may face. For this reason, we have included in the analysis and discussion risk factors with a significance of $\mathrm{p}<0.05$.

In conclusion, these results highlight the difficulty of managing medically complex and noncompliant patients who have ankle fractures requiring open reduction and internal fixation. All patients should be warned of potential woundrelated complications, particularly those who report high medical risk factors, including diabetes (particularly those with peripheral neuropathy); those taking medications that may affect wound-healing; and those with open fractures. With meticulous patient optimization and surgical technique, the risk of wound-related complications can be very low; in the present series, only $1.25 \%$ of patients required further surgical treatment of their wounds. We also believe that preoperative monitoring of swelling and delaying the time to surgery can diminish the risk of wound complications. However, our analysis of swelling was subjective, and we are unable to provide quantitative conclusions based on swelling from our data. This conjecture needs to be confirmed in a future randomized comparative study. While most risk factors cannot be controlled, postoperative care is an area of importance for woundhealing. The adherence to postoperative instructions should be emphasized, and the treating surgeon should remain vigilant to the potential effect that noncompliance may have on woundhealing after surgical intervention.

Adam G. Miller, MD

Andrew Margules, MD

Department of Orthopedics,

Thomas Jefferson University Hospital,

Curtis Building \#801, 1015 Walnut Street,

Philadelphia, PA 19107

Steven M. Raikin, MD

Rothman Institute, 925 Chestnut Street,

Philadelphia, PA 19107.

E-mail address: steven.raikin@rothmaninstitute.com 
The Journal of Bone \& Joint Surgery $\cdot$ Jbjs.org Volume 94-A • Number 22 - November 21, 2012
Risk Factors for Wound Complications After

Ankle Fracture Surgery

\section{References}

1. Salai M, Dudkiewicz I, Novikov I, Amit Y, Chechick A. The epidemic of ankle fractures in the elderly -is surgical treatment warranted? Arch Orthop Trauma Surg. 2000;120(9):511-3.

2. Pagliaro AJ, Michelson JD, Mizel MS. Results of operative fixation of unstable ankle fractures in geriatric patients. Foot Ankle Int. 2001 May;22(5):399-402.

3. Koval KJ, Zhou W, Sparks MJ, Cantu RV, Hecht P, Lurie J. Complications after ankle fracture in elderly patients. Foot Ankle Int. 2007 Dec;28(12):1249-55.

4. Leyes M, Torres R, Guillén P. Complications of open reduction and internal fixation of ankle fractures. Foot Ankle Clin. 2003 Mar;8(1):131-47, ix.

5. Flynn JM, Rodriguez-del Rio F, Pizá PA. Closed ankle fractures in the diabetic patient. Foot Ankle Int. 2000 Apr;21(4):311-9.

6. Blotter RH, Connolly E, Wasan A, Chapman MW. Acute complications in the operative treatment of isolated ankle fractures in patients with diabetes mellitus. Foot Ankle Int. 1999 Nov;20(11):687-94.

7. Wukich DK, Kline AJ. The management of ankle fractures in patients with diabetes. J Bone Joint Surg Am. 2008 Jul;90(7):1570-8.

8. Bibbo C, Lin SS, Beam HA, Behrens FF. Complications of ankle fractures in diabetic patients. Orthop Clin North Am. 2001 Jan;32(1):113-33.

9. SooHoo NF, Krenek L, Eagan MJ, Gurbani B, Ko CY, Zingmond DS. Complication rates following open reduction and internal fixation of ankle fractures. J Bone Joint Surg Am. 2009 May;91(5):1042-9.

10. Mak KH, Chan KM, Leung PC. Ankle fracture treated with the AO principle-an experience with 116 cases. Injury. 1985 Jan;16(4):265-72.

11. Lindsjö U. Operative treatment of ankle fracture-dislocations. A follow-up study of 306/321 consecutive cases. Clin Orthop Relat Res. 1985 Oct;(199):28-38.

12. Höiness $P$, Engebretsen $L$, Strömsöe $K$. Soft tissue problems in ankle fractures treated surgically. A prospective study of 154 consecutive closed ankle fractures. Injury. 2003 Dec;34(12):928-31.

13. Franz MG, Robson MC, Steed DL, Barbul A, Brem H, Cooper DM, Leaper D, Milner SM, Payne WG, Wachtel TL, Wiersema-Bryant L; Wound Healing Society. Guidelines to aid healing of acute wounds by decreasing impediments of healing. Wound Repair Regen. 2008 Nov-Dec;16(6):723-48.

14. Haidukewych GJ. Temporary external fixation for the management of complex intra- and periarticular fractures of the lower extremity. J Orthop Trauma. 2002 Oct; 16(9):678-85
15. Zalavras CG, Christensen T, Rigopoulos N, Holtom P, Patzakis MJ. Infection following operative treatment of ankle fractures. Clin Orthop Relat Res. 2009 Jul;467(7):1715-20. Epub 2009 Feb 19.

16. Wukich DK, Lowery NJ, McMillen RL, Frykberg RG. Postoperative infection rates in foot and ankle surgery: a comparison of patients with and without diabetes mellitus. J Bone Joint Surg Am. 2010 Feb;92(2):287-95.

17. Acello AN, Wallace GF, Pachuda NM. Treatment of open fractures of the foot and ankle: a preliminary report. J Foot Ankle Surg. 1995 Jul-Aug;34(4): 329-46.

18. Tho KS, Chiu PL, Krishnamoorthy S. Grade III open ankle fractures-a review of the outcome of treatment. Singapore Med J. 1994 Feb;35(1):57-8.

19. Carragee EJ, Csongradi JJ. Increased rates of complications in patients with severe ankle fractures following interinstitutional transfers. J Trauma. 1993 Nov;35(5):767-71.

20. Carragee EJ, Csongradi JJ, Bleck EE. Early complications in the operative treatment of ankle fractures. Influence of delay before operation. J Bone Joint Surg Br. 1991 Jan;73(1):79-82.

21. Breederveld RS, van Straaten J, Patka $P$, van Mourik JC. Immediate or delayed operative treatment of fractures of the ankle. Injury. 1988 Nov;19(6):436-8.

22. Bray TJ, Endicott M, Capra SE. Treatment of open ankle fractures. Immediate internal fixation versus closed immobilization and delayed fixation. Clin Orthop Relat Res. 1989 Mar;(240):47-52.

23. Joshi D, Singh D, Ansari J, Lal Y. Immediate open reduction and internal fixation in open ankle fractures. J Am Podiatr Med Assoc. 2006 Mar-Apr;96(2): 120-4.

24. Hulsker CC, Kleinveld S, Zonnenberg CB, Hogervorst M, van den Bekerom MP. Evidence-based treatment of open ankle fractures. Arch Orthop Trauma Surg. 2011 Nov;131(11):1545-53. Epub 2011 Jun 29.

25. Fong W, Acevedo J, Stone RG, Mizel MS. The treatment of unstable ankle fractures in patients over eighty years of age. Foot Ankle Int. 2007 Dec;28(12): 1256-9.

26. Bhandari M, Sprague S, Hanson B, Busse JW, Dawe DE, Moro JK, Guyatt GH. Health-related quality of life following operative treatment of unstable ankle fractures: a prospective observational study. J Orthop Trauma. 2004 Jul;18(6): 338-45. 